\title{
Review
}

\section{Overdiagnosis and overtreatment of breast cancer Progression of ductal carcinoma in situ: the pathological perspective}

\author{
J Louise Jones
}

Tumour Biology Laboratory, Institute of Cancer, Queen Mary's School of Medicine and Dentistry, Charterhouse Square, London, UK

Corresponding author: J Louise Jones, louise.j.jones@cancer.org.uk

Published: 21 April 2006

This article is online at http://breast-cancer-research.com/content/8/2/204

(c) 2006 BioMed Central Ltd

\begin{abstract}
Ductal carcinoma in situ (DCIS) is encountered much more frequently in the screening population compared to the symptomatic setting. The behaviour of DCIS is highly variable and this presents difficulties in choosing appropriate treatment strategies for individual cases. This review discusses the current data on the frequency and rate of progression of DCIS, the value and limitations of clinicopathological and biological variables in predicting disease behaviour and suggests strategies to develop more robust means of predicting progression of DCIS.
\end{abstract}

\section{Introduction}

Ductal carcinoma in situ (DCIS) is a neoplastic proliferation of cells within the ductal-lobular structures of the breast that has not penetrated the myoepithelial-basement membrane interface. In the symptomatic setting, DCIS accounts for $3 \%$ to $5 \%$ of cancers, although with the advent of population based screening it now represents approximately $20 \%$ of breast cancers detected $[1,2]$.

Epidemiological, histopathological and genetic studies have provided compelling evidence to support the concept that DCIS represents the precursor of invasive carcinoma in the majority of cases. In recent years, this 'model of progression' of breast cancer has been further refined with the recognition and characterisation of earlier precursor lesions, such as atypical ductal hyperplasia and the columnar cell lesions [3].

Because the aim of screening is to detect and treat disease in its early stages in order to prevent life-threatening disease, it could be argued that DCIS (and maybe even earlier lesions) represents the ideal target in a strategy to prevent invasive breast cancer.

This makes the assumption that all DCIS will inevitably progress to invasive carcinoma; however, much about the
Breast Cancer Research 2006, 8:204 (doi:10.1186/bcr1397)

behaviour of DCIS is still poorly understood because opportunities to study its natural history are limited. Over recent years, large clinical trials and follow-up on several important studies in which patients received diagnostic biopsy alone as treatment for their DCIS have provided further insight into the behaviour of this disease. The latter studies, which are biased towards lower-grade lesions, show that, untreated, up to $50 \%$ of DCIS lesions progress to invasive disease, and that the time for progression may be up to four decades $[4,5]$. Conversely, this also indicates that half of these lesions do not progress to invasive disease within a woman's lifetime. The challenge is to define better ways of quantifying the risk of progression for individual lesions in order to better tailor treatment decisions.

This review aims to present available data regarding the frequency and rate of progression of DCIS, the value and limitations of clinicopathological and biological characteristics in predicting progression and suggests future strategies to help more clearly define disease potential and inform treatment decisions.

\section{Clinicopathological variables and relation to progression}

DCIS is a heterogeneous disease varying in clinical presentation, morphology and behaviour. In an attempt to reflect this heterogeneity, several histopathological classification systems have been proposed. These are based on nuclear morphology, growth pattern, cytonuclear differentiation and the presence or absence of necrosis, in various combinations. Some of these classification systems provide poor reproducibility, thereby limiting their clinical practice [6]; however, others give more acceptable levels of reproducibility and have been shown to be clinically relevant. The Van Nuys classification system is based on nuclear grade and necrosis, yielding three subgroups of DCIS - non-high-grade without

$\mathrm{DCIS}=$ ductal carcinoma in situ 
necrosis, non-high-grade with necrosis and high-grade with or without necrosis - that show an association both with local recurrence and disease-free survival [7]. The classification system recommended by the National Coordinating Group for Breast Screening Pathology in the United Kingdom [8] classifies the disease into three groups on the basis of cytonuclear features, with low-grade DCIS being composed of small regular cells, often showing a cribriform or micropapillary architecture. High-grade DCIS is composed of large, pleomorphic cells with frequent mitotic figures, often having a solid architecture and associated necrosis. Intermediate-grade DCIS shows nuclear features intermediate between low- and high-grade DCIS. This classification system has also shown clinical relevance, with high-grade DCIS exhibiting a higher frequency of recurrence than lowgrade DCIS [9].

Careful analysis of large series of cases is essential to establish accurate information on disease behaviour. In the Van Nuys series of 866 DCIS patients, there were a total of 98 recurrences, 46\% invasive and 54\% non-invasive, with the probability of an invasive recurrence at 8 years calculated to be $6.4 \%$ and breast cancer-specific mortality to be $1.1 \%$ [10]. This correlates well with other studies reporting mortality rates of approximately $1.5 \%$ to $2.0 \%$ at 10 years [1].

Bijker and colleagues [11] followed up 775 cases of DCIS as part of a randomised clinical trial of breast conserving surgery for DCIS with or without radiotherapy. Recurrence was detected in 125 cases at a median follow-up of 5.4 years: 65 developed recurrent DCIS whilst 60 developed invasive breast cancer. In addition to histological type (nuclear grade, architecture and necrosis), other factors found to be related to the rate of recurrence included young patient age, mode of presentation with recurrence being more common in symptomatically detected lesions, extent of disease and margin status. Radiotherapy reduced the risk of recurrence compared to local excision alone, although recurrence was still high in patients with involved or not-stated margins, supporting previous studies emphasising the importance of margin status [12]. Interestingly, whereas intermediate- and high-grade DCIS showed significantly higher risk of recurrent $\mathrm{DCIS}$ compared to low-grade DCIS, the risk of recurrence as invasive disease was independent of DCIS grade. The outcome of the invasive disease differed markedly between the grades, however, with the risk of distant metastasis and death being significantly higher in recurrences secondary to high-grade DCIS [11]. It has previously been shown that the grade of DCIS corresponds to the grade of subsequent invasive carcinoma [13] and together with genetic studies showing different patterns of chromosomal alterations in DCIS and invasive carcinomas of different grades [14] these findings support the proposal that different grades of DCIS represent distinct disease entities that give rise to different pathways of disease evolution.
In view of these data, it could be argued that treatment of DCIS should focus particularly on preventing recurrence in high-grade DCIS, since this is most likely to progress to lifethreatening disease. However, several studies that report the long-term follow-up of patients with DCIS treated with biopsy alone have been important in highlighting the progression potential of lower-grade lesions.

Betsill and colleagues [15] reported on the outcome of 10 patients with low-grade DCIS treated with biopsy alone with a mean follow-up of 21.6 years, and found that 7 had developed invasive carcinoma at an average interval of 9.7 years (range of 7 to 30 years). In the most recent report from the long-term follow-up study of Page and colleagues [4] in which 28 women with small low-grade DCIS were treated with biopsy alone, 11 women developed invasive breast cancer (39.3\%). Of these, 7 were diagnosed within 10 years of their biopsy, 1 within 12 years and 3 over 23 to 42 years. Five of the 11 women who developed invasive carcinoma died of metastatic disease, with a mean follow-up of 31 years. In all cases, the invasive carcinoma developed in the same quadrant of the same breast that the biopsy had been taken, supporting the proposal that this represents disease progression rather than de novo disease.

Comparable findings come from the Nurses Health Study [5], which showed that of 13 patients with DCIS treated by biopsy alone, 10 developed recurrent disease: 6 developed invasive carcinoma and 4 developed recurrent in situ disease. Invasive carcinoma developed in patients with DCIS of all grades, including 2 of 6 with low-grade disease (at 5 and 18 years post-biopsy), 2 of 6 intermediate-grade DCIS (at 5 and 16 years post-biopsy) and 2 of 3 with high-grade DCIS (at 4 and 5 years post-biopsy). Taken together, these studies suggest that whilst progression to invasive disease is more rapid in high-grade DCIS, all grades have significant potential to progress.

A much lower recurrence rate was reported by Eusebi and colleagues [16], who found 11 of 80 women with DCIS treated with biopsy alone developed invasive carcinoma at a mean follow-up of 17.5 years. This is likely to reflect the inclusion of a proportion of cases categorised as pure clinging DCIS. In the report from Bijker and colleagues [11], an analysis of recurrence rate in relation to histological subtype of low-grade DCIS revealed that none of 59 cases of clinging DCIS developed recurrence, in contrast to low-grade cribriform lesions, which exhibited similar recurrence rates to high grade DCIS. To reflect this very low risk of recurrence and progression, clinging DCIS is now categorised under the heading of 'flat epithelial atypia', and this illustrates the value of such long-term studies to help refine diagnostic categories.

Biological variables and relation to prognosis Biological and genetic studies have aimed to refine the prediction of disease behaviour that can be achieved on the 
basis of pathological features alone. For many of these studies there are confounding factors that make interpretation complex, for example, the effect of disease extent and margin involvement.

Of the biological markers most extensively analysed, possibly one of the studies that most effectively controls for these factors is that of Cornfield and colleagues [17]. They analysed 151 cases of DCIS detected by mammography or as an incidental finding in a benign biopsy (suggesting limited disease extent); all were treated by breast conserving surgery alone and confirmed clear of margins. They found recurrence in 42 cases $(27.8 \%)$ between 11 and 97 months following surgery and analysed disease behaviour in relation to expression of oestrogen receptor, progesterone receptor, p53, Ki-67, HER2, bcl-2 and p21 in addition to conventional histopathological parameters. Whilst correlations between biological markers were noted (e.g., significant negative correlation between HER2 and oestrogen receptor, and between HER2 and bcl-2), none of the biological markers exhibited any association with disease recurrence, either in univariate or multivariate analysis [17].

Other workers have reported associations between some of these markers and disease recurrence. Ringberg and colleagues [18] found high $\mathrm{Ki}-67$, high p53 and reduced bcl-2 to be associated with recurrence in univariate analysis, although in multivariate analysis including tumour grade and growth pattern none reached statistical significance. This is largely in agreement with other reports that show a relationship between grade of $\mathrm{DCIS}$ and biological factors, such as p53 mutation [19].

Whilst these studies suggest that biological markers do not enhance the predictive power of histopathological factors, Barnes and colleagues [20] have recently reported the independent prognostic value of HER4 expression in DCIS. HER4 has been shown to modulate HER2 signalling leading to a reduction in cell proliferation [21]. In a retrospective analysis, this group showed that HER2 and HER3 expression was significantly associated with disease recurrence whereas HER4 expression was significantly less frequent in disease that recurred. Expression of HER4 in the absence of HER2 led to a highly significant disease-free survival advantage, and the prognostic significance of HER4 expression was maintained in multivariate analysis that included disease grade and margin status. Whether HER4 expression can contribute to the recognition of a subgroup of DCIS with better prognosis remains to be established and will require larger long-term studies and, in particular, greater numbers of lower grade lesions.

Another approach trying to define factors that will predict behaviour of DCIS has been to identify changes in gene or protein expression associated with the transition to invasive disease. Ma and colleagues [22] used laser capture microdissection of in situ and invasive disease followed by cDNA microarray analysis to generate a gene expression profile associated with disease progression. The major differences in gene expression profile were seen in relation to different grade invasive tumours rather than disease stage and generally disease of different stages from the same patient clustered more closely than stage-specific disease. One exception was in high grade DCIS where cluster analysis identified a set of 29 genes that was more highly expressed in the matched invasive component compared to the DCIS, suggesting they may be related to progression. The gene set included genes involved in cell cycle control, centrosomal function and DNA repair, and one gene, RRM2, which has been linked to metastatic potential. As yet, the value of such a gene profile has not been established, though data from similar studies comparing invasive and matched metastatic carcinomas suggest a common predictive gene signature could be elusive [23].

A recent report has proposed that the natural history of different DCIS subtypes relates to the pattern of development of DCIS within the breast lobe [24]. This hypothesis, based on extensive review of large two- and three-dimensional histological sections of DCIS, suggests that DCIS may appear as unifocal, multifocal or diffuse disease due to involvement of individual terminal ductal-lobular units, several distant terminal ductal-lobular units or involvement of larger ducts. They found that low-grade DCIS tends to be multifocal, intermediate grade unifocal and diffuse with duct neogenesis almost exclusively high grade and this has an impact on recurrence. This study also associated the diffuse neogenetic DCIS with expression of Tenascin- $C$ in the stroma, a reflection of the remodelling occurring in high grade disease. Such changes in the microenvironment may influence tumour progression. Alterations in the myoepithelial cell population and the stromal cells around DCIS have been described that could influence initiation of invasion [25-27]. However, further functional studies and analysis of larger case series will be required to determine the importance of such phenotypic changes.

\section{Conclusion}

The studies to date indicate that DCIS of all grades has the potential to progress, though high-grade lesions progress more rapidly than lower grade lesions and are more likely to lead to metastatic disease and death. Long-term follow-up studies have further stratified disease behaviour and led to the identification of lesions that exhibit significantly lower progression potential, such as flat cell atypia, and it is clear that these should be treated differently to DCIS. Given the different behaviour of low and high grade DCIS, both in terms of leading to life-threatening disease and in the time for evolution of the disease, it would appear warranted to tailor treatment more closely to disease type, with less aggressive therapy for low grade lesions. However, the challenge remains to accurately identify the small number of low-grade lesions likely to progress in order to provide the most 
This article is part of a review series on

Overdiagnosis and overtreatment of breast cancer, edited by Nick E Day, Stephen Duffy and Eugenio Paci.

Other articles in the series can be found online at

http://breast-cancer-research.com/articles/

review-series.asp?series=BCR_Overdiagnosis

appropriate treatment without over-treating the vast majority of patients with lesions that will be cured by breast conserving surgery alone.

Thus far, biological studies have not had a significant impact on predicting disease behaviour, although continued efforts, informed by functional (i.e., in vitro and animal models) and genetic studies, together with greater understanding afforded by studies of disease development as described in [24], and careful analysis of large clinical trials should help to better define disease behaviour and define distinct subgroups of DCIS with prognostic significance. Because the events being measured are rare, that is, recurrence, invasion and metastasis, particularly for low grade lesions, meaningful studies to test the predictive power of new approaches and biological markers will require analysis on large numbers of cases with follow-up, which is most likely to be achieved through material gained from clinical trials.

\section{Competing interests}

The author declares that they have no competing interests.

\section{References}

1. Ernster VL, Barclay J, Kerlikowske K, Wilkie H, Ballard-Barbash R: Mortality among women with ductal carcinoma in situ of the breast in the population-based surveillance, epidemiology and end results program. Arch Intern Med 2000, 160:953-958.

2. Van Dongen JA, Fentiman IS, Harris JR, Holland R, Peterse JL, Salvadori B, Stewart HJ: In situ breast cancer: the EORTC consensus meeting. Lancet 1989, ii:25-27.

3. Schnitt SJ: Flat epithelial atypia - classification, pathologic features and clinical significance. Breast Cancer Res 2003, 5: 263-268.

4. Saunders ME, Schuyler PA, Dupont WD, Page DL: The natural history of low grade ductal carcinoma in situ of the breast in women treated by biopsy only revealed over 30 years of longterm follow-up. Cancer 2005, 103:2481-2484.

5. Collins LC, Tamimi RM, Baer HJ, Connoly JL, Colditz GA, Schnitt $\mathrm{SJ}$ : Outcome of patients with ductal carcinoma in situ untreated after diagnostic biopsy. Results from the Nurses' Health Study. Cancer 2005, 103:1778-1784.

6. Van Dongen JA, Holland R, Peterse JL, Fentiman IS, Lagios MD, Millis RR, Recht A: Ductal carcinoma in situ of the breast: second EORTC consensus meeting. Eur J Cancer 1992, 28: 626-629.

7. Silverstein MJ, Lagios MD, Craig PH, Waisman JR, Lewinsky BS, Colburn WJ, Poller DN: A prognostic index for ductal carcinoma in situ of the breast. Cancer 1996, 77:2267-2274.

8. National Coordinating Group for Breast Screening Pathology: Guidelines for Pathology Reporting in Breast Cancer Screening. 3rd edition. Sheffield: NHSBSP Publications; 2005.

9. Badve S, A'Hern RP, Ward AM, Millis RR, Pinder SE, Ellis IO, Gusterson BA, Sloane JP: Prediction of local recurrence of ductal carcinoma in situ of the breast using five histological classifications: a comparative study with long follow-up. Hum Pathol 1998, 29:915-923.
10. Skinner KA, Silverstein MJ: The management of ductal carcinoma in situ of the breast. Endocr Relat Cancer 2001, 8:33-45.

11. Bijker N, Peterse JL, Julien J-P, Fentimen IS, Duval C, Di Palma S, Simony-Lafontaine J, de Mascarel I, van de Vijver M: Risk factors for recurrence and metastasis after breast conserving therapy for ductal carcinoma in-situ: analysis of European organisation for research and treatment of cancer trial 10853. $J$ Clin Oncol 2001, 19:2263-2271.

12. Silverstein MJ, Lagios MD, Groshen S, Waisman JR, Lewinsky BS, Martino S, Gamagami P, Colburn WJ: The influence of margin width on local control of ductal carcinoma in situ of the breast. N Eng J Med 1999, 340:1455-1461.

13. Millis RR, Ryder K, Fentiman IS: Ductal carcinoma in situ and prognosis in invasive mammary carcinoma. Breast Cancer Res Treat 2004, 84:197-198.

14. Simpson PT, Reis-Filho JS, Gale T, Lakhani SR: Molecular evolution of breast cancer. J Patho/ 2005, 205:248-254.

15. Betsill WLJ, Rosen PP, Lieberman PH, Robbins GF: Intraductal carcinoma. Long tern follow-up after treatment by biopsy alone. J Am Med Assoc 1978, 239:1863-1867.

16. Eusebi V, Feudale E, Foschini MP, Micheli A, Conti A, Riva C, Di Palma S, Rilke F: Long-term follow-up of in-situ carcinoma of the breast. Semin Diag Pathol 1994, 11:223-235.

17. Cornfield DB, Palazzo JP, Schwartz GF, Goonewardene SA, Kovatich AJ, Chervoneva I, Hyslop T, Schwarting R: The prognostic significance of multiple morphologic features and biologic markers in ductal carcinoma in situ of the breast. A study of a large cohort of patients treated with surgery alone. Cancer 2004, 100:2317-2327.

18. Ringberg A, Anagnostaki L, Anderson H, Idvall I, Ferno M: Cell biological factors in ductal carcinoma in situ (DCIS) of the breast - relationship to ipsilateral local recurrence and histopathological characteristics. Eur J Cancer 2001, 37:15141522.

19. Done SJ, Eskandarain S, Bull S, Redson M, Andrulis IL: p53 missense mutation in microdissected high grade ductal carcinoma in situ of the breast. J Natl Cancer Inst 2001, 93: 700-704.

20. Barnes NLP, Khavari S, Boland GP, Cramer A, Knox WF, Bundred NJ: Absence of HER4 expression predicts recurrence of ductal carcinoma in situ of the breast. Clin Cancer Res 2005, 11:2163-2168.

21. Sartor $\mathrm{Cl}$, Zhou H, Kozlowska E, Guttridge K, Kawata E, Caskey L, Harrelson J, Hynes N, Ethier S, Calvo B, Earp HS: HER4 mediates ligand dependent antiproliferative and differentiation responses in human breast cancer cells. Mol Cell Biol 2001, 21:4265-4275.

22. Ma XJ, Salunga R, Tuggle JT, Gaudet J, Enright E, McQuary P, Payette T, Pistone M, Stecker K, Zhang BM, et al:: Gene expression profiles of breast cancer progression. Proc Natl Acad Sci USA 2003, 100:5974-5979.

23. Weigelt B, Wessels LFA, Bosma AJ, Glas AM, Nuyten DSA, He YD, Dai H, Peterse JL, van't Veer LJ: No common denominator for breast cancer lymph node metastasis. Br J Cancer 2005, 93:924-932.

24. Tot T: DCIS, cytokeratins and the theory of the sick lobe. Virchows Arch 2005, 447:1-8.

25. Adams M, Jones JL, Walker RA, Pringle JH, Bell SC: Altered Tenascin- $C$ isoform expression in invasive and pre-invasive breast cancer. Cancer Res 2002, 62:3289-3297.

26. Allinen M, Beroukhim R, Cal LI, Brennan C, Lahti-Domenici J, Huang H, Porter D, Hu M, Chin L, Richardson A, et al:: Molecular characterisation of tumour microenvironment in breast cancer. Cancer Cell 2004, 6:17-32.

27. Nielsen BS, Rank F, Lopez JM, Balbin M, Vizoso F, Lund LR, Dano $\mathrm{K}$, Lopez-Otin C: Collagenase-3 expression in breast myofibroblasts as a molecular marker of transition of ductal carcinoma in-situ lesions to invasive ductal carcinomas. Cancer Res 2001, 61:7091-7100. 